\title{
Electronic compressibility and charge imbalance relaxation in cuprate superconductors
}

\author{
Ch. Helm ${ }^{\text {a,* }}$, L.N. Bulaevskii ${ }^{\mathrm{b}}$, D.A. Ryndyk ${ }^{\mathrm{c}}$, J. Keller ${ }^{\mathrm{c}}$, S. Rother ${ }^{\text {d }}$, Y. Koval ${ }^{\mathrm{d}}$, \\ P. Müller ${ }^{\mathrm{d}}$, R. Kleiner ${ }^{\mathrm{e}}$ \\ a Institut für Theoretische Physik, ETH Hönggerberg, 8093 Zürich, Switzerland \\ ${ }^{\mathrm{b}}$ Los Alamos National Laboratory, Los Alamos, NM 87545 \\ ${ }^{\mathrm{c}}$ Institut für Theoretische Physik, Universität Regensburg, D-93040 Regensburg, Germany \\ ${ }^{\mathrm{d}}$ Physikalisches Institut III, Universität Erlangen-Nürnberg,D-91058 Erlangen, Germany \\ ${ }^{\mathrm{e}}$ Physikalisches Institut, Universität Tübingen, D-72076 Tübingen,Germany
}

\begin{abstract}
In the material $\mathrm{SmLa}_{1-x} \mathrm{Sr}_{x} \mathrm{CuO}_{4-\delta}$ with alternating intrinsic Josephson junctions we explain theoretically the relative amplitude of the two plasma peaks in transmission by taking into account the spatial dispersion of the Josephson Plasma Resonance in $c$ direction due to charge coupling. From this and the magnetic field dependence of the plasma peaks in the vortex solid and liquid states it is shown that the electronic compressibility of the $\mathrm{CuO}_{2}$ layers is consistent with a free electron value. Also the London penetration depth $\lambda_{a b} \approx 1100 \AA$ near $T_{c}$ can be determined. The voltage response in the $I V$-curve of a $\mathrm{Bi}_{2} \mathrm{Sr}_{2} \mathrm{CaCu}_{2} \mathrm{O}_{8}$ mesa due to microwave irradiation or current injection in a second mesa is related to the nonequilibrium charge imbalance of quasiparticles and Cooper pairs and from our experimental data the relaxation time $\sim 100$ ps is obtained.
\end{abstract}

Key words: intrinsic Josephson effect, optical spectroscopy, transport, charge imbalance, $\mathrm{Bi}_{2} \mathrm{Sr}_{2} \mathrm{CaCu}_{2} \mathrm{O}_{8}, \mathrm{SmLa}_{1-x} \mathrm{Sr}_{x} \mathrm{CuO}_{4-\delta}$ PACS: $74.25 . \mathrm{Gz}, 74.50 .+\mathrm{r}, 74.72 . \mathrm{h}$

The theoretical analysis of optical and transport properties of intrinsic Josephson junctions in high- $T_{c}$ superconductors allows to extract key microscopic parameters of the $\mathrm{CuO}_{2}$-layers, such as the electronic compressibility and the charge imbalance relaxation rate, which are hard to obtain otherwise.

In optical transmission or reflectivity experiments (cf. Fig. 1a) the Josephson Plasma Resonance (JPR) of Cooper pairs oscillating between the layers, creates a peak in the loss function $L(\omega)$ (e.g. in TBCCO [1]). Recently, in the novel material $\mathrm{SmLa}_{1-x} \mathrm{Sr}_{x} \mathrm{CuO}_{4-\delta}$ with alternating junctions two plasma peaks were observed with a high ratio of their amplitudes $[2,3]$. This can only be explained, if the spatial dispersion of the JPR

\footnotetext{
* Corresponding author. Tel.: $++41-(0) 1-6332573$; fax: ++41 -
} (0)1-633 1115; email: helm@phys.ethz.ch

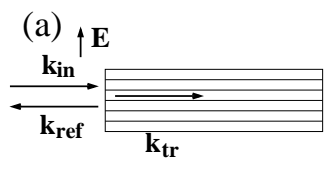

(c)

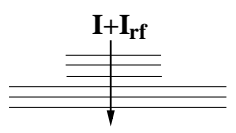

(b)

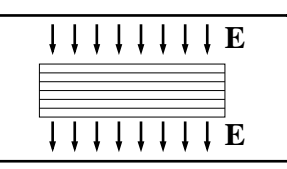

(d)

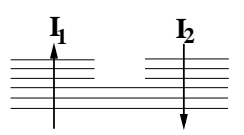

Fig. 1. Geometry for measuring (a) the reflectivity with parallel incidence, (b) the microwave absorption in a cavity, (c) Shapiro steps, and (d) dc-voltage response on dc-current injection

in $c$ direction due to charge fluctuations of the superconducting condensate, is taken into account, see Fig. 2 and Ref. [4]. Using the data in $[2,3]$ we find that the electronic compressibility of the layers is consistent with a free electron value. This corresponds to a $c$ axis disper- 


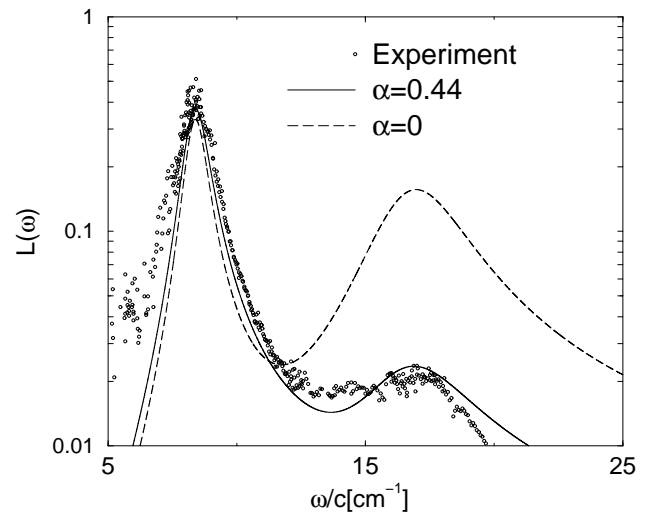

Fig. 2. Experimental data [3] together with our calculated loss function $L(\omega)$ for $\sigma_{1}=r \sigma_{2}=0.12 \epsilon_{0} \omega_{0,1} / 4 \pi, r=\omega_{0,1}^{2} / \omega_{0,2}^{2}$ (solid: $\alpha=0.44, r=0.38, \omega_{0,1} / c=7.2 \mathrm{~cm}^{-1}, \epsilon_{0}=18$; dashed: $\left.\alpha=0, r=0.24, \omega_{0,1} / c=8.3 \mathrm{~cm}^{-1}, \epsilon_{0}=13\right)$.

sion $\sim \alpha$ of the (bare) JPR frequencies, $\omega_{l}^{2}=\omega_{0, l}^{2}(1+$ $\left.\alpha k_{z}^{2}\right), l=1,2$ with $\alpha=\left(\epsilon_{0} / 4 \pi e s\right)(\partial \mu / \partial \rho) \approx 0.4\left(\epsilon_{0}\right.$ background dielectric constant, $s=6.3 \AA$ interlayer distance, $\mu$ chemical potential, $\rho 2 \mathrm{~d}$ charge density). This result is confirmed by the magnetic field dependence of the plasma peaks [5], using the dependence of the critical current densities, which is well known in the vortex liquid state and has been newly derived for the vortex solid [6]. The parameter $\alpha$ is not only relevant to understand the coupled dynamics of stacks of intrinsic Josephson junctions, e.g. for $\mathrm{THz}$ applications, but might also provide an important input for microscopic theories of high- $T_{c}$-superconductivity. Beyond that, the $c$-axis quasiparticle conductivity $\sigma_{1} \sim 4-$ $10(\Omega \mathrm{m})^{-1}$ and the London penentration depth $\lambda_{a b}=$ $1100 \AA$ at $T=10 \mathrm{~K}$ (near $T_{c}$ ) parallel to the layers can be accurately determined. Our theory for $L(\omega)$ quantitatively improves Ref. [7] by taking into account the different conductivities $\sigma_{l}$ in the junctions in accordance with their different current densities and the influence of the discrete atomic structure. Our results are in principle also applicable for microwave absorption experiments in a cavity (Fig. 1b).

From a theoretical point of view the inclusion of the wavevector dependence of the dielectric function $\epsilon(\omega, \mathbf{k})$ poses a nontrivial problem and requires to go beyond the conventional Fresnel theory [8]. Generally, for systems with spatial dispersion (e.g. phonons [9]), multiple eigenmodes with different group velocities are excited in the crystal and at certain extremal frequencies, where the group velocity vanishes, the atomic structure of the crystal enters explicitly in optical properties. The possibility to stop light dynamically by affecting the JPR in an external magnetic field might serve as a building block for a future magneto-optical device, e.g. to store photonic qubits [8].

Further, we perform two different transport exper- iments on $\mathrm{Bi}_{2} \mathrm{Sr}_{2} \mathrm{CaCu}_{2} \mathrm{O}_{8}$, in order to investigate nonequilibrium effects beyond the coupling $\alpha$ [10]: (1) In 2-point measurements of the $I V$-curves in the presence of high-frequency irradiation $\left(I_{\mathrm{rf}}\right)$ of frequency $f$ a shift of the voltage of Shapiro steps of $\sim 3 \%$ from the canonical value $V_{s}=\hbar f / 2 e$ has been observed due to the resistance at the $N S$-contact (Fig. 1c, first discussed in [11]). (2) In the $I V$-curves of double-mesa structures an influence of the dc voltage $V_{1}$ measured at one mesa on the dc quasiparticle current $I_{2}$ injected into the other mesa is detected (Fig. 1d). Both effects can be explained by charge-imbalance on the superconducting layers between resistive and superconducting junctions, where Cooper pairs and quasiparticles are transformed into each other. With the help of a recently developed theory [12] we get the charge imbalance relaxation time as $\approx 70-450$ ps (depending on the sample).

In conclusion, the optical spectroscopy of the Josephson plasma resonance in $\mathrm{SmLa}_{1-x} \mathrm{Sr}_{x} \mathrm{CuO}_{4-\delta}$ suggests a free electronic compressibility of the superconducting $\mathrm{CuO}_{2}$-layers and a London penetration depth $\lambda_{a b}=$ $1100 \AA$ near $T_{c}$. From the analysis of the resistive state in the presence of microwave irradiation or current injection a charge imbalance relaxation time of the order $\sim 100$ ps is obtained.

The authors thank for fruitful discussions with G. Blatter, M. Dressel, B. Gorshunov, D. van der Marel, A. Pimenov, and Z.-X. Shen and acknowledge financial support of the Swiss NCCR "MaNEP", the U.S. DOE, the German DFG and the Bayerische Forschungsstiftung.

\section{References}

[1] V. K. Thorsmølle et al., Optics Letters 26 (2001) 1292; Physica B 312 (2002) 84

[2] D. Dulić et al., Phys.Rev.Lett. 86 (2001) 4144; H. Shibata, Phys.Rev.Lett. 86 (2001) 2122; T. Kakeshita et al. Phys.Rev.Lett. 86 (2001) 4140.

[3] B. Gorshunov et al., to be published.

[4] Ch. Helm, L.N. Bulaevskii, E.M. Chudnovsky, M.P. Maley, Phys.Rev.Lett. 89 (2002) 057003.

[5] A. Pimenov et al., Phys.Rev.Lett. 87 (2001) 7003.

[6] L.N. Bulaevskii, C. Helm, Phys.Rev.B 66 (2002) 174505.

[7] D. van der Marel et al., Phys.Rev.B 64 (2001) 24530.

[8] L.N. Bulaevskii, Ch. Helm, A.R. Bishop, M.P. Maley, Europhys. Lett. 58 (2002) 415; Ch. Helm, L.N. Bulaevskii, Phys.Rev.B 66 (2002) 094514.

[9] I. Kaelin, C. Helm, G. Blatter, physics/0303046

[10] S. Rother, Y. Koval, P. Müller, R. Kleiner, D.A. Ryndyk, J. Keller, Ch. Helm, Phys.Rev.B 67 (2003) 024510.

[11] C. Helm et al., Physica C 362 (2001) 43. 
[12] D. A. Ryndyk, J. Keller, C. Helm, J.Phys.: Cond. Mat. 14 (2002) 815 